\title{
EVIDENCIA HEMATOLÓGICA Y SEROLÓGICA DE Ehrlichia spp EN PROPIETARIOS DE CANINOS DOMÉSTICOS CONANTECEDENTES DE EHRLICHIOSIS EN LIMA METROPOLITANA
}

\author{
Hematological and Serological Evidence of Ehrlichia SPP in OWNers of \\ Domestic Canines with History of Ehrlichiosis in Lima
}

\author{
Luis Barrios A. ${ }^{1}$, Olga Lí E. ${ }^{1,4}$, Francisco Suárez A..$^{2}$, Alberto Manchego S. ${ }^{3}$, \\ Luis Hoyos S. ${ }^{1}$
}

\section{Resumen}

\begin{abstract}
El presente estudio tuvo por objetivo determinar la seropositividad a Ehrlichia canis mediante la prueba de inmunofluorescencia indirecta (IFI) en pacientes humanos, sus parámetros hematológicos y su asociación con las variables edad, sexo, nivel de contacto con perros y antecedentes de exposición a garrapatas. Se evaluaron 91 sujetos sin distinción de género, edad o condición socioeconómica cuyos perros tenían historia de erlichiosis dentro de los seis meses previos al muestreo. El muestreo se realizó entre febrero y mayo de 2010. La evaluación hematológica mostró ausencia de pacientes positivos y $15.4 \%$ de pacientes sospechosos (presencia de corpúsculos de inclusión en células mononucleares y ausencia de trombocitopenia). La frecuencia de pacientes seropositivos mediante IFI fue de $14.3 \%$. No se encontró diferencia estadística por efecto de las variables en estudio. Los hallazgos confirman la exposición a E. canis en propietarios de caninos domésticos con antecedentes de ehrlichiosis en Lima Metropolitana.
\end{abstract}

Palabras clave: Ehrlichia canis, humanos, hematología, corpúsculos de inclusión, inmunofluorescencia indirecta

\section{Abstract}

The aim of this study was determine the frequency of seropositivity to a Ehrlichia canis in human subjects by indirect inmunofluorescent test (IFI), hematological changes and its association with risk factors such as age, gender, level of contact with dogs, and tick exposure. Ninety-one samples were collected from patients without reference to gender, age and socioeconomic status, but whose dogs had history of ehrlichiosis within six months to the sampling. The study was conducted between February and May 2010. Hematology results revealed absence of positive cases and $15.4 \%$ of suspected cases

\footnotetext{
${ }^{1}$ Laboratorio de Patología Clínica, ${ }^{2}$ Laboratorio de Medicina Veterinaria Preventiva, ${ }^{3}$ Laboratorio de Microbiología y Parasitología Veterinaria, Facultad de Medicina Veterinaria, Universidad Nacional Mayor de San Marcos, Lima

${ }^{4}$ E-mail:olgalie@hotmail.com
} 
(presence of inclusion bodies in mononuclear cells and absence of thrombocytopenia). Frequency of IFI seropositives was $14.3 \%$. No statistical difference was found due to the effect of the variables under evaluation. The results confirm exposure to E. canis in dog owners with previous record of ehrlichiosis in Metropolitan Lima.

Key words: Ehrlichia canis, humans, hematology, inclusion bodies, indirect immunofluorescent test

\section{INTRODUCCIÓN}

Una de las enfermedades más comunes transmitidas por garrapatas en perros domésticos es la ehrlichiosis canina, cuyo agente causal principal es Ehrlichia canis, transmitida al animal por medio de la picadura de la garrapata marrón del perro (Rhipicephalus sanguineus). Las ehrlichiosis son consideradas como enfermedades zoonóticas emergentes y se reconocen dos tipos de enfermedades: la ehrlichiosis monocítica humana, causada por $E$. chaffeensis (Paddock y Childs, 2003) y la ehrlichiosis granulocítica humana, causada por Anaplasma phagocytophilum. En el Perú, se han reportado únicamente dos agentes infecciosos pertenecientes a la familia Anaplasmataceae, que son E. canis y A. marginale, causantes de la ehrlichiosis monocítica canina y la anaplasmosis bovina, respectivamente (Chavera et al., 1982; Vinasco et al., 2007).

Los primeros reportes de E. canis como potencial agente zoonótico datan de 1986, año en que fue descrito el primer caso de infección humana (Paddock y Childs, 2003). Los síntomas asociados a la enfermedad varían de acuerdo a la edad y estado inmune del paciente (Harkess et al., 1991); siendo fiebre, cefalea, fatiga y anorexia los más importantes (Rohrbach et al., 1990). A nivel de laboratorio, los principales signos de sospecha son leucopenia, anemia, linfopenia, trombocitopenia, hiperproteinemia/ hipergamaglobulinemia y elevación de las enzimas hepáticas (Paddock y Childs, 2003).
Estos hallazgos, tan variables e inespecíficos, hacen que esta enfermedad sea considerada en el diagnóstico diferencial de pacientes febriles con antecedentes de exposición o picadura de garrapatas (Anaya et al., 2006); sin embargo, en el Perú aún no se han detectado pacientes positivos a la enfermedad con sintomatología clínica compatible.

Los primeros estudios de ehrlichiosis canina en el país reportaron seroprevalencias de $16.5 \%$ en Lima Metropolitana (Adrianzén, 2002) y de $76 \%$ en Sullana (San Miguel, 2006). En América del Sur, existen evidencias serológicas de ehrlichiosis humana en Brasil (1.2\%) (da Costa et al., 2006), Argentina (14.3) (Ripoll et al., 1999), Chile (10.5\%) (López et al., 2003) y de infección por $E$. canis en Venezuela (30\%) (Pérez et al., 2006).

Se dispone de cuatro reportes de ehrlichiosis humana en el Perú. En el 2008, en Lima Metropolitana, se detectó mediante la prueba de inmunofluorescencia indirecta (IFI) que el $20.1 \%$ y el $26.1 \%$ de pacientes resultaron seropositivos a $E$. chaffeensis y E. canis, respectivamente, mientras que el $13.3 \%$ resultaron positivos a ambas bacterias (O. Li, Lima, datos no publicados). $\mathrm{Al}$ año siguiente, se realizó un estudio similar, encontrándose 21.1 y $23.3 \%$ de pacientes seropositivos a E. chaffeensis y E. canis, respectivamente, utilizando la misma prueba (O. Li, Lima, datos no publicados). En Ancash (2009) se encontró 9.2\% de seropositivos mediante la prueba de IFI (Anaya et al., 2009); y en ese mismo año, se hallaron seroprevalencias de $25 \%$ en Piura, $23 \%$ en 
Cusco y $3 \%$ en Iquitos y Lima (Moro et al., 2009).

Por estas razones, se hizo un estudio para evaluar, mediante el análisis hematológico y serológico, la presencia de esta enfermedad en propietarios de caninos domésticos en Lima Metropolitana.

\section{Materiales y Métodos}

\section{Lugar de Estudio y Personas}

Se recolectaron muestras a 91 personas de 20 distritos de Lima Metropolitana: Ate Vitarte (2), Carabayllo (4), Callao (6), Cercado de Lima (3), Comas (4), Chaclacayo (1), Chorrillos (2), La Molina (2), La Victoria (1), Lurín (3), Pachacamac (4), San Bartolo (1), San Isidro (1), San Luis (3), Santa Anita (6), San Juan de Lurigancho (34), Villa el Salvador (3), San Martín de Porres (2), San Juan de Miraflores (5) y Surco (4). El estudio se llevó a cabo entre febrero y mayo de 2010.

Las personas que se incluyeron en el estudio fueron seleccionadas sin distinción de género, edad, condición socioeconómica o estado de salud; sin embargo, debían poseer por lo menos un perro en casa, y que se encontrase cursando o haya cursado con ehrlichiosis (aguda o crónica) dentro de los últimos seis meses. Todas las personas respondieron un cuestionario que incluía datos personales y clínico-epidemiológicos. En el caso de los niños, los padres llenaron los cuestionarios. El tipo de muestreo fue de carácter no probabilístico en base a sujetos voluntarios, siguiendo las normas éticas de la Declaración de Helsinki, modificada en 1983.

\section{Muestras y Análisis de Laboratorio}

Se recolectó $8 \mathrm{ml}$ de sangre periférica dividida en dos partes: $2 \mathrm{ml}$ con EDTA con anticoagulante y $6 \mathrm{ml}$ sin anticoagulante, para la obtención de suero. La obtención de sangre venosa con jeringa se realizó según las indicaciones recomendadas por el Instituto Nacional de Salud (INS, 2005) del Perú, y se hizo posterior a las seis semanas de la aceptación de los pacientes en el estudio.

Las muestras para hematología y estudio de capa flogística se procesaron de inmediato en el Laboratorio de Patología Clínica, mientras que las muestras para serología se centrifugaron y los sueros resultantes fueron conservados en congelación a $-20{ }^{\circ} \mathrm{C}$ hasta su procesamiento en el Laboratorio de Microbiología y Parasitología Veterinaria. Ambos laboratorios forman parte de la Facultad de Medicina Veterinaria, Universidad Nacional Mayor de San Marcos, Lima.

A nivel hematológico de determinó el recuento de eritrocitos $\left(\mathrm{x} 10^{6} / \mu \mathrm{l}\right)$ y leucocitos $\left(\mathrm{x} 10^{3} / \mu \mathrm{l}\right)$ utilizando la cámara de Neubauer, hemoglobina $(\mathrm{g} / \mathrm{dl})$ por el método de la cianometahemoglobina, hematocrito (\%) por el método del microhematocrito, y el recuento diferencial (\%) y recuento plaquetario (il) utilizando la tinción Wright.

Además, se hicieron las siguientes determinaciones:

- Nivel de infectividad ocasionado por Ehrlichia spp, calculado tanto en valores relativos como absolutos. Para el cálculo del nivel de infectividad relativo se empleó la fórmula: (número de células infectadas $/ 100$ células contadas en el recuento diferencial) x 100. Las células infectadas eran células mononucleares con inclusiones intracitoplasmáticas. El nivel de infectividad absoluto se calculó multiplicando el valor relativo por el número total de leucocitos.

- Máximo número de inclusiones por célula: Se determinó a partir de la observación del frotis teñido con tinción Wright. Sólo se tomó el valor de la célula con mayor número de inclusiones.

- Presencia de linfocitos granulares, linfocitos atípicos y apoptosis. 
Cuadro 1. Resultados del examen hematológico de 14 pacientes sospechosos de ehrlichiosis (Lima, 2010)

\begin{tabular}{|c|c|c|c|c|c|}
\hline Variable & Sexo & Media & $\begin{array}{c}\text { Desviación } \\
\text { estándar }\end{array}$ & Rango & $\begin{array}{l}\text { Valores } \\
\text { normales }\end{array}$ \\
\hline \multirow{2}{*}{ Eritrocitos $\left(\times 10^{6} / \mu \mathrm{l}\right)$} & $\mathrm{F}$ & 4.6 & 0.3 & $4.1-4.9$ & $(3.8-4.8)$ \\
\hline & M & 5.0 & 0.3 & $4.6-5.5$ & $(4.5-5.5)$ \\
\hline \multirow{2}{*}{ Hemoglobina (g/dl) } & $\mathrm{F}$ & 12.6 & 1.7 & $10.1-14.6$ & $(12-16)$ \\
\hline & M & 14.1 & 1.2 & $12.3-15.9$ & $(14-18)$ \\
\hline \multirow{2}{*}{ Hematocrito (\%) } & $\mathrm{F}$ & 40.0 & 2.0 & $36-42$ & $(37-47)$ \\
\hline & M & 43.0 & 2.5 & $40-47$ & $(41-53)$ \\
\hline $\mathrm{N}^{\circ}$ de leucocitos $\left(\mathrm{x} 10^{3} / \mu \mathrm{l}\right)$ & & 7.75 & 0.26 & $4.0-13.4$ & $(4.5-11.5)$ \\
\hline - Neutrófilos (\%) & & 52.0 & 6.6 & $37.0-62.0$ & $(55-70)$ \\
\hline - Linfocitos $(\%)$ & & 41.0 & 6.4 & $29.0-54.0$ & $(17-45)$ \\
\hline - Monocitos (\%) & & 2.0 & 2.0 & $0.0-6.0$ & $(2-8)$ \\
\hline - Eosinófilos (\%) & & 4.0 & 2.5 & $1.0-9.0$ & $(1-4)$ \\
\hline - Basófilos (\%) & & 0.8 & 1.0 & $0.0-3.0$ & $(0.2-1.2)$ \\
\hline - Neutrófilos $\left(\times 10^{3} / \mu 1\right)$ & & 4.1 & 1.6 & $2.2-7.3$ & $(2.5-7.5)$ \\
\hline - Linfocitos $\left(\times 10^{3} / \mu 1\right)$ & & 3.1 & 1.1 & $1.5-5.5$ & $(1.3-4.0)$ \\
\hline - Monocitos $\left(\times 10^{3} / \mu \mathrm{l}\right)$ & & 0.2 & 0.1 & $0.0-0.4$ & $(0.15-0.9)$ \\
\hline - Eosinófilos $\left(\times 10^{3} / \mu 1\right)$ & & 0.3 & 0.2 & $0.1-0.8$ & $(0.06-0.5)$ \\
\hline - Basófilos $\left(\times 10^{3} / \mu 1\right)$ & & 0.01 & 0.08 & $0.0-0.3$ & $(0.01-0.15)$ \\
\hline - Plaquetas $\left(\times 10^{3} / \mu \mathrm{l}\right)$ & & 266 & 35.9 & $204-324$ & $(150-400)$ \\
\hline
\end{tabular}

Cuadro 2. Distribución de pacientes hematológicamente sospechosos según los resultados de la prueba de IFI para ehrlichiosis (Lima, 2010)

\begin{tabular}{cccc}
\hline \multirow{2}{*}{ IFI } & \multicolumn{2}{c}{$\begin{array}{c}\text { Presencia de } \\
\text { corpúsculos de } \\
\text { inclusión }\end{array}$} & \multirow{2}{*}{ Total } \\
\cline { 2 - 3 } & Sí & No & \\
\hline Sí & 2 & 11 & 13 \\
No & 12 & 66 & 78 \\
\hline Total & 14 & 77 & 91 \\
\hline
\end{tabular}

- Presencia de corpúsculos de inclusión compatibles con Ehrlichia spp en capa flogística.

Las personas no trombocitopénicas, sin corpúsculos de inclusión en leucocitos, pudiendo presentar otras citopenias (anemia, leucopenia), fueron consideradas como negativos, y aquellas personas sin trombocitopenia con presencia de corpúsculos de inclusión en leucocitos y que presentaron otras citopenias fueron consideradas como sospechosas. Sólo se consideraron positivos a ehrlichiosis a las personas con trombocitopenia y presencia de corpúsculos de inclusión en leucocitos. 

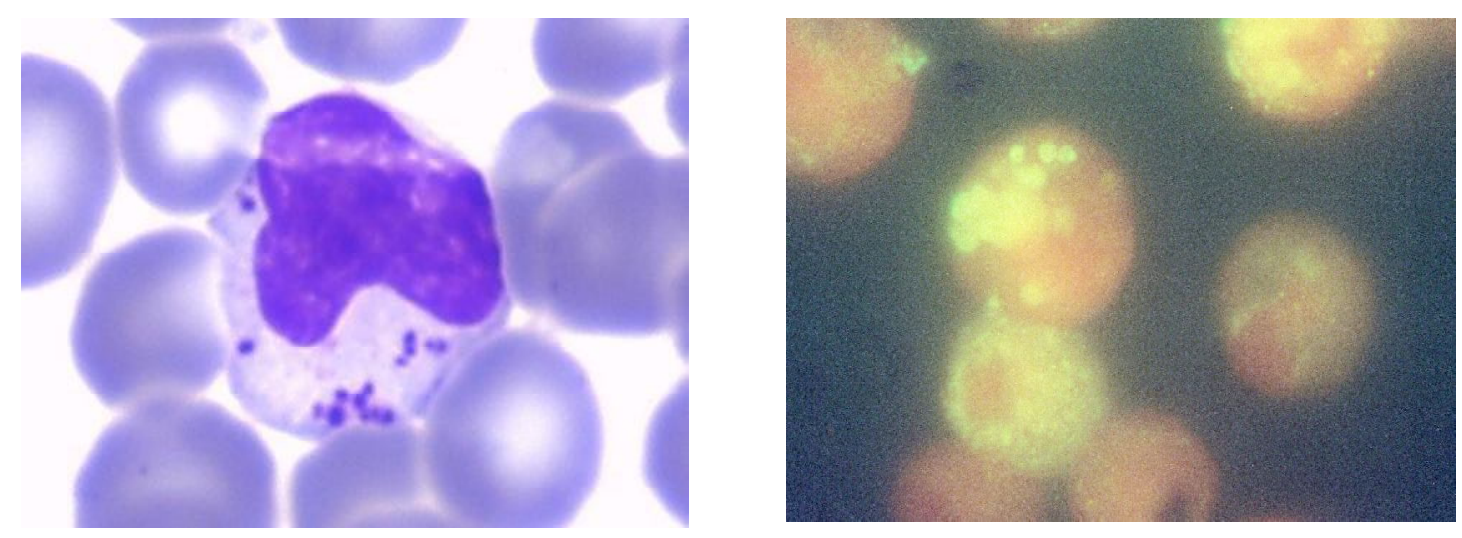

Figura 1. (a) Linfocito con presencia de corpúsculos de inclusión azurófilos en su citoplasma. Nótese la disposición irregular y morfología heterogénea de las inclusiones, así como las deformaciones del citoplasma y del núcleo. Tinción Wright. 100X; (b) Suero positivo a Ehrlichia canis por inmunofluorescencia indirecta (IFI), dilución (1:50). 40X.

Se utilizó la prueba de inmunofluorescencia indirecta (IFI) para la detección de anticuerpos contra Ehrlichia canis según el procedimiento utilizado por Gutiérrez (2008). Se utilizó una dilución del suero de 1:50.

\section{Análisis Estadístico}

Se empleó estadística descriptiva (media, desviación estándar, rangos) y tablas de frecuencia. Asimismo, se utilizó la prueba de Chi cuadrado con un nivel de confianza del 95\% para evaluar la asociación estadística entre las variables edad, sexo, nivel de contacto con perros y antededentes de exposición a garrapatas en relación a la presencia de anticuerpos contra $E$. canis. Se utilizó el software estadístico SPSS 16 y MINITAB 15.

\section{Resultados}

Ningún paciente resultó positivo por medio del examen hematológico, mientras que $15.4 \%$ resultaron sospechosos a la enfermedad al encontrarse corpúsculos de inclusión en linfocitos, pero sin mayores cambios en relación a los valores normales referidos por la OMS (Cuadro 1) (OMS, 2012). El nivel de infectividad resultó desde 1 hasta $14 \%$ en valores relativos, mientras que los valores absolutos fueron de 85 a 938 células/ $\mu 1$. El máximo número de inclusiones fue de 48 en un linfocito, sin encontrar mórulas de Ehrlichia spp en las personas sospechosas (Fig. 1a).

Mediante la serología se encontró $14.3 \%$ de pacientes seropositivos, de los cuales $30.8 \%$ eran hombres $(4 / 13)$ y $69.3 \%$ eran mujeres (9/13). Asimismo, 53.8 y $46.2 \%$ eran menores y mayores de 40 años de edad, respectivamente. Por otro lado, el 92.3\% (12/13) de los pacientes estuvieron expuestos a garrapatas.

No se apreció diferencia estadística a la prueba de Chi cuadrado para las variables en estudio (edad, género, nivel de contacto con perros y antecedentes de exposición a garrapatas) en relación a la presencia de anticuerpos contra E. canis. Tampoco se encontró una relación significativa entre pacientes que resultaron sospechosos y los serológicamente positivos (Cuadro 2). 


\section{Discusión}

La evaluación hematológica reflejó ausencia de pacientes positivos; es decir, no hubo casos con trombocitopenia y presencia de corpúsculos de inclusión en leucocitos. Este resultado indica dos situaciones posibles. La primera posibilidad era que los pacientes no padecían la fase aguda de la enfermedad aunque hayan sido expuestos a garrapatas y hayan tenido un alto nivel de contacto con sus mascotas; ya que esta fase se caracteriza por durar de 8 a 21 días, tiempo mucho menor a las seis semanas o más que hubo entre el inicio de alguna sintomatología inespecífica y la toma de muestra. La segunda posibilidad fue que los pacientes presentasen alguna otra enfermedad con sintomatología inespecífica que curse con alteraciones hematológicas como anemia (hallada en el $26.4 \%$ de los pacientes).

Por otro lado, si bien no se hallaron pacientes hematológicamente positivos, se tuvo $15.4 \%$ de pacientes sospechosos con linfocitos invadidos por corpúsculos de inclusión. Estos resultados son similares a los observados en estudios previos (O. Li, Lima, datos no publicados), donde la frecuencia de muestras sanguíneas con corpúsculos de inclusión basófilos en linfocitos fue de $11.1 \%$. Es probable que estos individuos estuvieran cursando la fase subclínica de la enfermedad, ya que en esta fase se normalizan muchas alteraciones, principalmente anemia y trombocitopenia, pero persistiendo los corpúsculos de inclusión desde la fase aguda, aunque en menor cantidad (reportado según el nivel de infectividad y el número de inclusiones por célula).

El nivel de infectividad indica la cantidad de células infectadas tanto en valores relativos como en valores absolutos. El 14\% encontrado en el presente estudio fue considerado como muy alto, dado que el total de células mononucleares infectadas suele ser inferior al 1\%, con tendencia a disminuir conforme evoluciona la enfermedad (Cowell et al., 1988; Gilbert et al., 1992). Asimismo, el máximo número de 48 inclusiones por célula infectada podría significar una gran susceptibilidad de las células frente a posibles patógenos intracelulares oportunistas, debido a la inhibición masiva de los mecanismos de defensa asociado a la elevada cantidad de bacterias en el citoplasma.

En relación a los hallazgos hematológicos secundarios (linfocitos atípicos, linfocitos granulares, apoptosis y cambios tóxicos), la presencia de linfocitos atípicos fue similar al estudio de Hamilton et al. (2004). Estos linfocitos atípicos probablemente representen células $\mathrm{T}$ gamma/delta (Caldwell et al., 1996) o células NK. Sin embargo, cabe indicar que estos hallazgos fueron similares a los encontrados en pacientes hematológicamente negativos.

En cuanto al $14.3 \%$ de pacientes seropositivos (Fig. 1b), es importante señalar que la prueba de IFI puede mostrar reacción cruzada con especies ehrlichiales del mismo genogrupo, como E. chaffeensis y E. ewingii (Dumler et al., 2001). Se ha reportado incluso casos de reacción cruzada con especies de otros genogrupos (Neorickettsia helmintoeca y Anaplasma phagocytophilum).

Los resultados de seroprevalencia fueron mayores al 9.2\% encontrado en Ancash (Anaya et al., 2009); sin embargo, no existen estudios de seroprevalencia de ehrlichiosis en caninos ni se ha realizado la detección del agente causal en esa localidad, por lo que no se podría inferir la posibilidad de una diferencia entre ambas localidades en base a estos aspectos. Los hallazgos locales (O. Li, Lima, datos no publicados) difieren de los resultados del presente estudio, probablemente por el tipo de población evaluada ya que los pacientes, en su mayoría, ejercían actividades veterinarias.

La casuística de ehrlichiosis monocítica humana es mayor en pacientes que superan los 40 años de edad (Paddock y Childs, 2003) $\mathrm{y}$ en pacientes del género masculino (Stone 
et al., 2005); sin embargo, estas variables no fueron estadísticamente significativas en el presente estudio. El $69.2 \%$ de pacientes mujeres seropositivas podría deberse a que en las familias de los pacientes encuestados, las mujeres eran las que se quedaban con las mascotas en el hogar aumentando el riesgo de exposición a garrapatas; no obstante, esta diferencia no fue estadísticamente significativa.

La ausencia de significancia estadística entre los grupos con y sin evidencia de exposición a garrapatas podría interpretarse en base a una exposición reciente de la mayoría de pacientes seropositivos, toda vez que una exposición reciente a garrapatas infectadas con especies ehrlichiales no permitiría el desarrollo de anticuerpos detectables mediante la prueba de IFI; además, la picadura de garrapatas puede pasar desapercibida y, por lo tanto, no haberse registrado en la ficha personal de cada paciente evaluado. Por último, si bien hay estudios que revelan que el contacto con perros no parece ser un factor de riesgo para la infección en el hombre (Harkess et al., 1989), e incluso hay estudios que revelan que perros pertenecientes a pacientes con ehrlichiosis no presentaron evidencia serológica de infección canina reciente (Rohrbach et al., 1990). Hay otros estudios que afirman la participación del perro como factor de riesgo debido a su densidad poblacional, alto contacto físico con las personas y hábitats comunes para muchas especies de garrapatas (Paddock y Childs, 2003).

\section{Conclusiones}

- En Lima Metropolitana, al menos el $14.3 \%$ de propietarios de caninos domésticos con antecedentes de ehrlichiosis presentan anticuerpos que reaccionan contra Ehrlichia canis; sin embargo, no evidencian positividad a la enfermedad mediante la evaluación hematológica aunque presentan alteraciones hematológicas compatibles con la enfermedad.
- Las variables edad, género, exposición a garrapatas y nivel de contacto con perros no estuvieron asociadas a la presencia de anticuerpos contra E. canis en propietarios de caninos domésticos con antecedentes de ehrlichiosis en Lima Metropolitana.

\section{Literatura Citada}

1. Adrianzen J. 2002. Seroprevalencia de la dirofilariosis y ehrlichiosis canina en los distritos de Chorrillos, La Molina y San Juan de Miraflores. Tesis de Médico Veterinario. Lima: Univ Nacional Mayor de San Marcos. 68 p.

2. Anaya E, Canelo L, Céspedes M, García M, Fuentes L, Tapia R, Troyes L. 2006. Etiología del síndrome febril agudo en la provincia de Jaén, Perú, 2004-2005. Rev Peru Med Exp Salud Pública 23(1): 5-11.

3. Anaya E, Morón C, Jaramillo K, Mendoza L, Román R. 2009. Evidencia serológica de ehrlichiosis humana en Ancash, Perú. Rev Peru Med Exp Salud Pública 26: 54-57.

4. Caldwell CW, Everett ED, McDonald G, Yesus YW, Roland WE, Huang HM. 1996. Apoptosis of gamma/delta T cells in human ehrlichiosis. Am J Clin Pathol 105: 640-646.

5. Chavera A, Viera F, Samamé H. 1982. Ehrlichiosis canina en el Perú. En: Anales VII Congreso Nacional de Ciencias Veterinarias. Ica.

6. Cowell RL, Tyler RD, Clinkenbeard KD, Meinkoth JH. 1988. Ehrlichiosis and polyarthritis in three dogs. J Am Vet Med Assoc 192: 1093-1095.

7. da Costa PS, Valle LM, Brigatte ME, Greco DB. 2006. More about human monocitotropic ehrlichiosis in Brazil: serological evidence of nine new cases. Braz J Infect Dis 10: 7-10.

8. Dumler JS, Barbet AF, Bekker CP, Dasch GA, Palmer GH, Ray SC, Rikihisa Y, Rurangirwa FR. 2001. Reorganization of genera in the familia 
Rickettsiaceae and Anaplasmataceae in the order Rickettsiales: unification of some especies of Ehrlichia with Anaplasma, Cowdria with Ehrlichia and Ehrlichia with Neorickettsia, descriptions of six new especies combinations and designation of Ehrlichia equi an 'HGE agent' as subjective synonyms of Ehrlichia phagocyitophila. Int J Syst Evol Microbiol 51:2145-2165.

9. Gilbert S, Clercx C, Henroteaux M. 1992. L'ehrlichiose canine. Ann Méd Vét 136: 505-510.

10. Gutiérrez V. 2008. Estudio comparativo entre el método de coloración de Wright y prueba de ELISA para el diagnóstico de ehrlichiosis canina en la ciudad de San Pedro Sula, Honduras. Tesis de Médico Veterinario. Guatemala: Univ. San Carlos de Guatemala. 65 p.

11. Hamilton $K S$, Stansaert S, Kinney MC. 2004. Characteristic peripheral blood findings in human ehrlichiosis. Mod Pathol 17: 512-517.

12. Harkess JR, Ewing SA, Crutcher JM, Kudlac J, McKee G, Istre GR. 1989. Human ehrlichiosis in Oklahoma. J Infect Dis 159: 576-579.

13. Harkess JR, Ewing SA, Brumit T, Mettry CR. 1991. Ehrlichiosis in children. Pediatrics 87: 199-203.

14. [INS] Instituto Nacional de Salud. 2005. Manuel de procedimientos de laboratorio en técnicas básicas de hematología. Serie de Normas Técnicas $\mathrm{N}^{\circ} 40.87 \mathrm{p}$.

15. López J, Rivera M, Concha JC, Gatica S, Loeffeholz M, Barriga O. 2003. Ehrlichiosis humana en Chile, evidencia serológica. Rev Med Chile 131: 67-70.
16. Moro P, Shah J, Li O, Gilman R, Harris N, Moro M. 2009. Serologic evidence of human ehrlichiosis in Peru. Am J Trop Med Hyg 80: 242-244.

17. [OMS] Organización Mundial de la Salud. 2012. Valores Internacionales. Disponible en: http://www.sld.cu/ galerias/pdf/sitios/hematologia/ valores_normales_adultos.pdf

18. Paddock C, Childs J. 2003. Ehrlichia chaffeensis: a prototypical emerging pathogen. J Clin Microbiol 1: 37-65.

19. Pérez M, Bodor M, Zhang C, Xiong Q, Rikihisa Y. 2006. Human infection with Ehrlichia canis accompanied by clinical signs in Venezuela. Ann N Y Acad Sci 1078: 110-117.

20. Ripoll CM, Remondegui CE, Ordonez G, Arazamendi R, Fusaro H, Hyman MJ, et al. 1999. Evidence of rickettsial spotted fever and ehrlichial infections in a subtropical territory of Jujuy, Argentina. Am J Trop Med Hyg 61: 350-354.

21. Rohrbach B, Harkess J, Ewing $S$, Kudlac J, Garry L, Mc Kee G, Istre G. 1990. Epidemiologic and clinical characteristics of persons with serologic evidence of $E$. canis infection. Am $\mathrm{J}$ Public Health 80: 442-445.

22. San Miguel CS. 2006. Prevalencia de Ehrlichia canis en caninos de la provincia de Sullana. Tesis de Médico Veterinario. Lima: Univ Alas Peruanas. 51 p.

23. Stone JH, Dierberg K, Aram G, Dumler JS. 2005. Human monocytic ehrlichiosis. JAMA 292: 2263-2270.

24. Vinasco J, Li O, Alvarado A, Díaz D, Hoyos L, Tabacchi L, Sirigireddy K, et al. 2007. Molecular evidence of a new strain of Ehrlichia canis from South America. J Clin Microbiol 45: 2716-2719. 